\title{
DELIVERING SOCIAL TELEREHABILITATION SERVICES
}

\author{
Gilberto Marzano
}

Velta Lubkina

\section{Lorita Rizakova}

Rezeknes Augstskola, Latvia

\begin{abstract}
Telerehabilitation is an emerging method of delivering rehabilitation services, which uses information and communication technologies to minimize distance and time barriers.

Telerehabilitation is often considered a specialization of the wide field of tele-medicine; most of telerehabilitation services fall into three categories: clinical assessment (patient's functional abilities in his or her environment), diagnosis and clinical therapy.

Researches have recently underlined the potential of social media, mobile phones, and the Internet in general for improving mental health, supporting positive outcomes on addiction issues, sexual health, and homelessness.

This paper analyses the issues and implications tied to the development of social telerehabilitation services in Latvia, and reports on the first step of National science program VPP INOSOCTEREHI, a new three years multidisciplinary project on social rehabilitation, which is conducted by four Latvian Universities, and focuses on the use of mobile technologies in rehabilitation scope.
\end{abstract}

Keywords: mobile technologies in rehabilitation, new media and health-care services, social rehabilitation, telerehabilitation services.

\section{What is social telerehabilitation}

The World Health Organization describes rehabilitation for people with disabilities as a process aimed at enabling them to reach and maintain their optimal physical, sensory, intellectual, psychological and social functional levels. Rehabilitation provides disabled people with the tools they need to attain independence and self-determination. ${ }^{17}$

Rehabilitation cannot be restricted to curative medicine, in the strict sense of medical body medicine. Medical rehabilitation involves intensively trained clinicians, specialized facilities, expert coordination, and different allied health professionals, such as physiotherapists, psychologists, occupational therapists, and social workers. In fact, medical care doesn't necessarily end with the patients discharge from the hospital. It may help patients recovering their capabilities or becoming independent at home. Rehabilitation related to individuals' social sphere is calledsocial rehabilitation. It includes services such as rehabilitation nursing, physiotherapy, occupational therapy, speech and language therapy, audiology, dietetics, prosthetics and orthotics, podiatry,

\footnotetext{
${ }^{17}$ http://www.who.int/topics/rehabilitation/en/; accessed 09/02/15.
} 
clinical psychology, art therapy, music therapy and social work. The border line between clinical and social rehabilitation is not well defined, and this fact can explain the failure of social scientists and policy makers to consider medical rehabilitation distinct from community-based efforts and general medical care (Haig, 2013).

Telerehabilitation ${ }^{18}$ is the use of information and communication technologies to deliver rehabilitation services over a distance. There is a clear link of telerehabilitation to the field of telecare, and the term telerehabilitation is often used as a synonym of tele-medicine and usually refers to equipment and services that provide continuous, automatic and remote monitoring of care needs, emergencies and lifestyle changes. Figure 1 shows the interaction between telemedicine, telecare and telerehabilitation.

Nowadays, telerehabilitation encompasses a wide range of applications which use new technologies to trigger human responses, control equipment at distance, assist patients, minimize or treat disabilities, cure physical and social diseases. In fact, decreasing time and mobility expenses, computer technologies save costs in the delivery of rehabilitation services, especially for those who are at risk of disabilities associated with stroke, heart disease, diabetes, arthritis, Alzheimer and other forms of mental and physical pathologies.

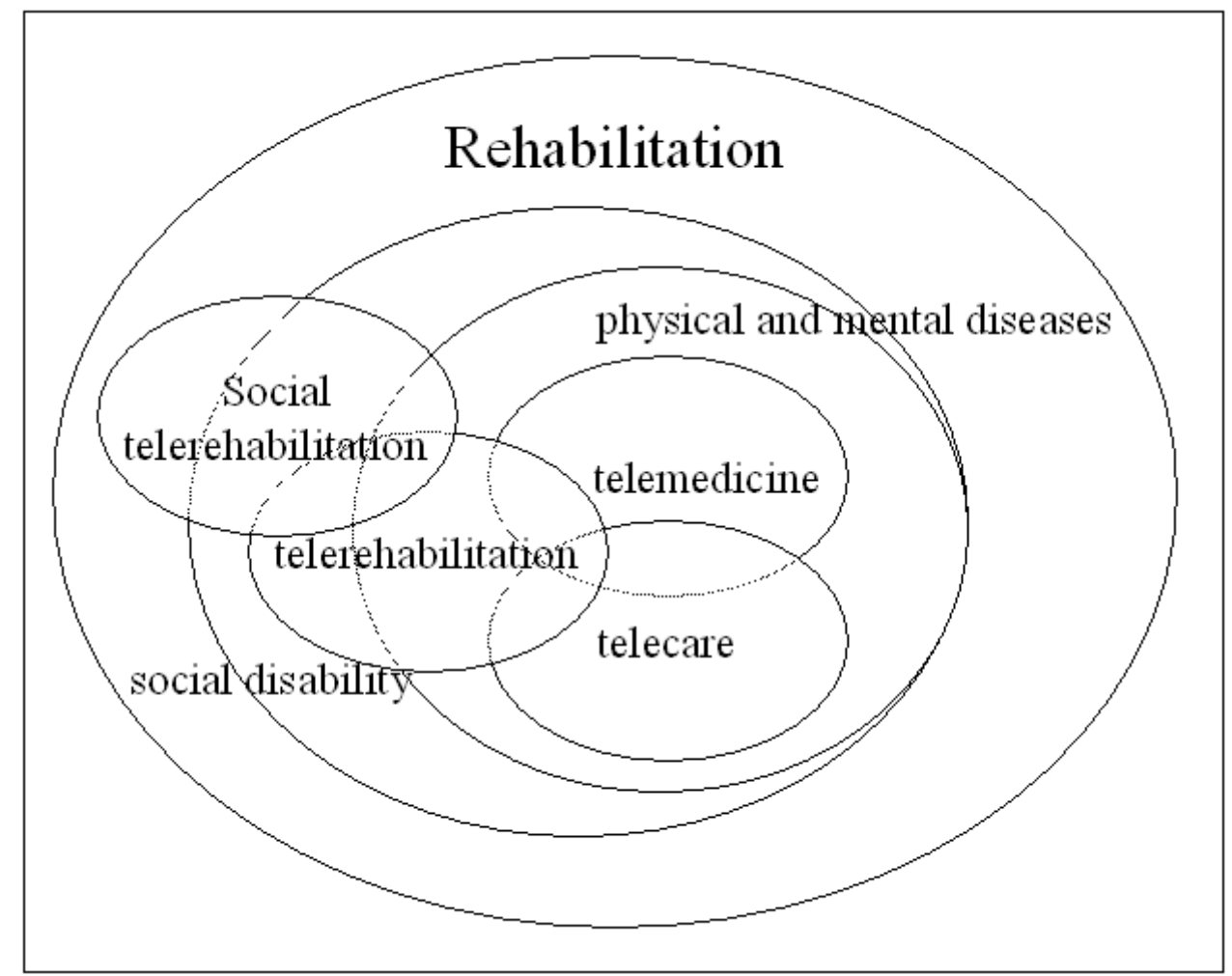

Figure 1. Interactions between telemedicine, telecare and telerehabilitation

\footnotetext{
${ }^{18}$ The term telerehabilitation first came to prominence in 1997 when the USADepartment of Education's National Institute on Disability and Rehabilitation Research issueda set of proposed priorities for the new Rehabilitation Engineering Research Center in the areaof what was called "tele-rehabilitation" (Winters, 2002).
} 
Current literature confirms the extent of telerehabilitation in a variety of areas, and shows the advantages and effectivity in expanding the quality of, and access to, rehabilitation services for many types of patients in both rural and urban areas (Rogante et. al., 2010; Hailey et al, 2011; Gorst et al., 2014; Di Cerbo et al., 2015).

Telerehabilitation is often considered a specialization of the wide field of tele-medicine, and most of telerehabilitation services fall into three categories: clinical assessment (the patient's functional abilities in his or her environment), diagnosis and clinical therapy.

Recently, researchers and practitioners argue the importance of social telerehabilitation services for patients, caregivers and public institutions, e. g. services for children suffering from physical handicaps and emotional disturbances (Nilsson \& Nilsson, 2011a, 2011b), for alcoholics (Simpson et al., 2005; Rose et al., 2012), and for patients with bipolar disorder (Osmani et al. 2013).

Research has underlined the potential for social media, mobile phones, and the Internet in general to improve mental health and physical health, treat addictions, and also help individuals experiencing homelessness (Freedman et. al., 2006; Luxton et al., 2011; Rice et al., 2012).

The scope of social telerehabilitation services faces challenges that set it apart from the broader telemedicine and telehealth arenas. It has been observed that one such challenge is that rehabilitation is often provided across both acute medical and community settings, often with different funding structures and rehabilitation protocols in place (Hill, 2010).

However, we are persuaded that social telerehabilitation is something more than the merely use of information and communication technology to support social rehabilitation in creating social rehabilitation services at distance. It should include the changes caused by rehabilitation distance services. Accordingly, a specific scope of social telerehabilitation is to make compliant rehabilitation technology with the social context, this is the health-care, family and working environments.

This view is one of the pillars of a research programme on social telerehabilitation, recently started in Latvia.

\section{The Latvian National science programme VPP INOSOCTEREHI}

National science programme VPP INOSOCTEREHI is a new three-year multidisciplinary project on social rehabilitation, conducted by four Latvian Universities, ${ }^{19}$ which investigates on the use of mobile technologies in rehabilitation scope.

\footnotetext{
${ }^{19}$ The universities involved in the research program are Rezeknes Augstskola, Latvia University, Technical University of Riga, Liepaja University; the project is leaded by Rezeknes Augstskola.
} 
In its first year of research activity, VPP INOSOCTEREHI project focuses on telerehabilitation solutions for balance disorder, aiming to broaden balance disorder prevention, and support diagnosis and treatments. Data previously acquired by Personality Socialization Research Institute (PSRI) of Rezeknes University using BioSway equipment (Baranauskienè et al., 2013), a portable balance system for testing and training patients, will be integrated with a new survey, and a Knowledge Platform will be realized. It will enclose and integrate competences, expertise and tools from different disciplinary fields (social pedagogy, special education, computer science, engineering, physiotherapy).

VPP INOSOCTEREHI research is principally driven by two goals:

1. extending people access to rehabilitation services, through the cutting of mobility costs;

2. increasing the quality of rehabilitation services, introducing new forms of prevention and monitoring in the health-care services.

In Latvia, rehabilitation care is currently provided by dedicated rehabilitation state hospitals and rehabilitation centers, while only a very small portion of the hospital sector is privately owned.

Despite many positive developments, Latvia health care services still don't meet the EU standards. Just like the other ex-soviet countries, in Latvia, until a few years ago (2009), life expectancy remained more than six years below the EU average of 79.8, principally because the objective of reducing cardiovascular mortality failed. After 2008, economic crisis reduced the financial resources available for health care, and the integration of care across providers still remains limited. In 2011, public spending on health in Latvia was about $3.7 \%$ of GDP, causing it to be one of the EU's highest rates of private, out-of-pocket health care expenditure. Personal insurance provides a mixture of complementary and supplementary coverage. It routinely covers those health care services and/or prescription drugs that are not statutorily financed. Patients' private expenditure on health care constituted $40 \%$ of total health financing, bringing total spending to $6.6 \%$ of GDP, which remains under the EU average for public health care funding (Terauda et al., 2014).

However, positive changes are in act, and new development strategies have been elaborated by the Ministry of Health, which emphasize the importance of patient centered health care, encouraging healthy lifestyle and physical activities, promoting healthy eating habits and aiming to the use of mobile technologies for implementing innovative solutions. ${ }^{20}$

A pressure into creating telerehabilitation services come from the newly introduced home care service for the chronically ill, which is based on cooperation between general practitioners and home care providers. ${ }^{21}$

\footnotetext{
${ }^{20}$ See the site of the Ministry of health (http://www.vm.gov.lv/en/; accessed 09/02/15).

${ }^{21}$ Commission for the Assessment of Health Condition and Working Ability, provides the possibility for patients to receive complex and problem-oriented care based on a rehabilitation plan written by a general practitioner or other medical specialist.
} 
Rehabilitation at home is now an available practice, it is based on a rehabilitation plan developed by physical medicine and rehabilitation specialists (Mitenbergs et al., 2012).

Inpatient rehabilitation is provided at the National Rehabilitation Centre and at several multi-profile hospitals. It consists of a range of services supplied by a multidisciplinary rehabilitation team. There exists long-term medical rehabilitation programmes for patients with chronic functional limitations; patients' functional conditions are monitored at regular intervals (at least once a year) and that necessary different rehabilitation services are coordinated with other medical professionals, family doctors and municipalities' social services.

The National Health System pays for rehabilitation services if patients have a referral from the appropriate specialist, who also has to develop a medical rehabilitation plan, including the aims, technologies and conditions of completion of rehabilitation.

\section{The research framework of VPP INOSOCTEREHI programme}

VPP INOSOCTEREHI research programme is developed along two dimensions, one horizontal, and the other vertical.

The horizontal dimension concerns the definition of the social rehabilitation context in terms of services, actors and regulations. The situation in Latvia is analyzed and compared with that of European countries, in particular with that of Baltic countries. The aim is to find potential applications of new technologies in the various sectors of social rehabilitation, identify common elements in terms of services typologies, needs to be met, skills and specializations, educational programmes, tools and basic requirements, etc. The analysis should provide the basement for the definition of a general telerehabilitation business model. It should be used for the construction of sectorial services in order to create economies of scale and integrated social rehabilitation services, as well as improve their quality. A crucial goal of the research programme is the definition of an educational programme to be included in the higher education curriculum, inasmuch many different disciplines and skills contribute to the design, and implement of telerehabilitation services (medicine, psychiatry, psychology, physiotherapy, social pedagogy, computer science, economics and management, etc.).

The vertical dimension concerns the comparative analysis of the solutions about balance disorder, a complex disturbance that affects many people worldwide. The research programme aims at verifying the feasibility of smartphone applications in balance deficits scope. The balance disorder is analyzed according to three fields of application: prevention, diagnosis and treatment. The available commercial solutions are analyzed and compared, and the possibility of using smartphones to support all or part of the existing solutions and to develop new applications will be investigated. 
SOCIETY. INTEGRATION. EDUCATION. Volume IV

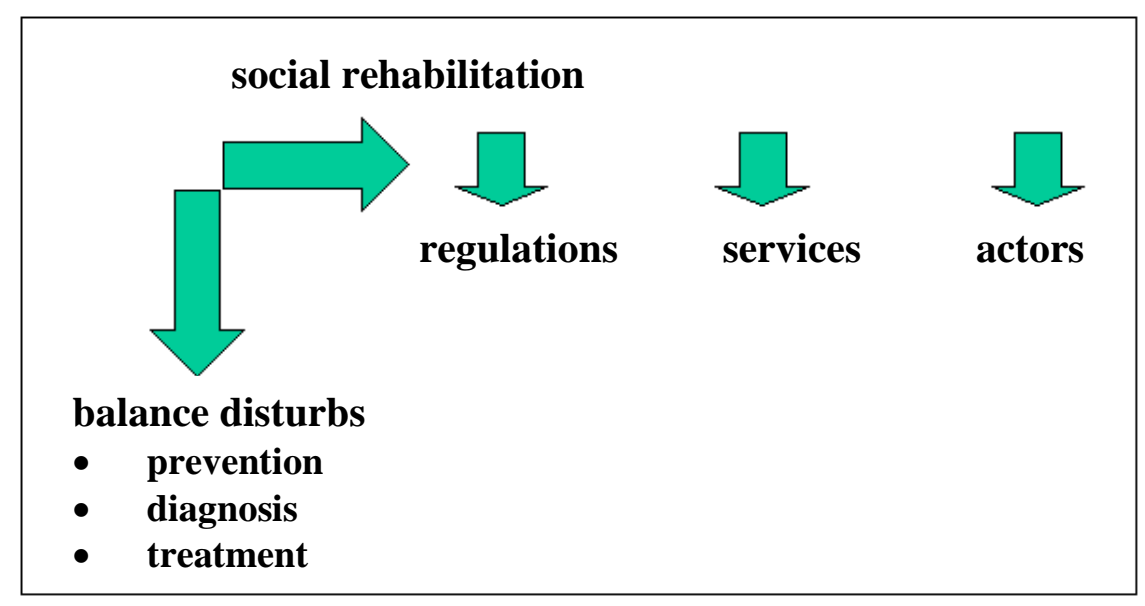

Figure 2. The research framework of VPP INOSOCTEREHI programme

The report on telerehabilitation in Scotland (Hill, 2010), has been one of the principal reference for defining our horizontal research. It identified opportunities and gave suggestions and recommendations in specific sectors, such as pulmonary rehabilitation, stroke rehabilitation, cognitive rehabilitation for people living with acquired brain injury, motor neuron disease, etc.

\section{Telerehabilitation of balance disorder}

Many people suffer from dizziness or imbalance during their lifetime. Human balance system involves a complex set of sensorimotor-control system (Figure 3), which includes sensory input from vision (sight), proprioception (touch), and the vestibular system (motion, equilibrium, spatial orientation). Problems with balance occur when there is a disruption in any of the vestibular, visual, or proprioceptive systems. The interlacing feedback mechanism which regulates the balance control can be disrupted by damage to one or more components through injury, disease, or aging process (Shumway-Cook \& Woollacott, 1995). A person can fill disoriented if his/her sensory input sources (eyes, muscles and joints) are in conflict with one another.

This brings about that balance dysfunction can be caused by problems in any one or a combination of the contributing systems. Accordingly, most balance patients travel a long and frustrating road before finding help. 


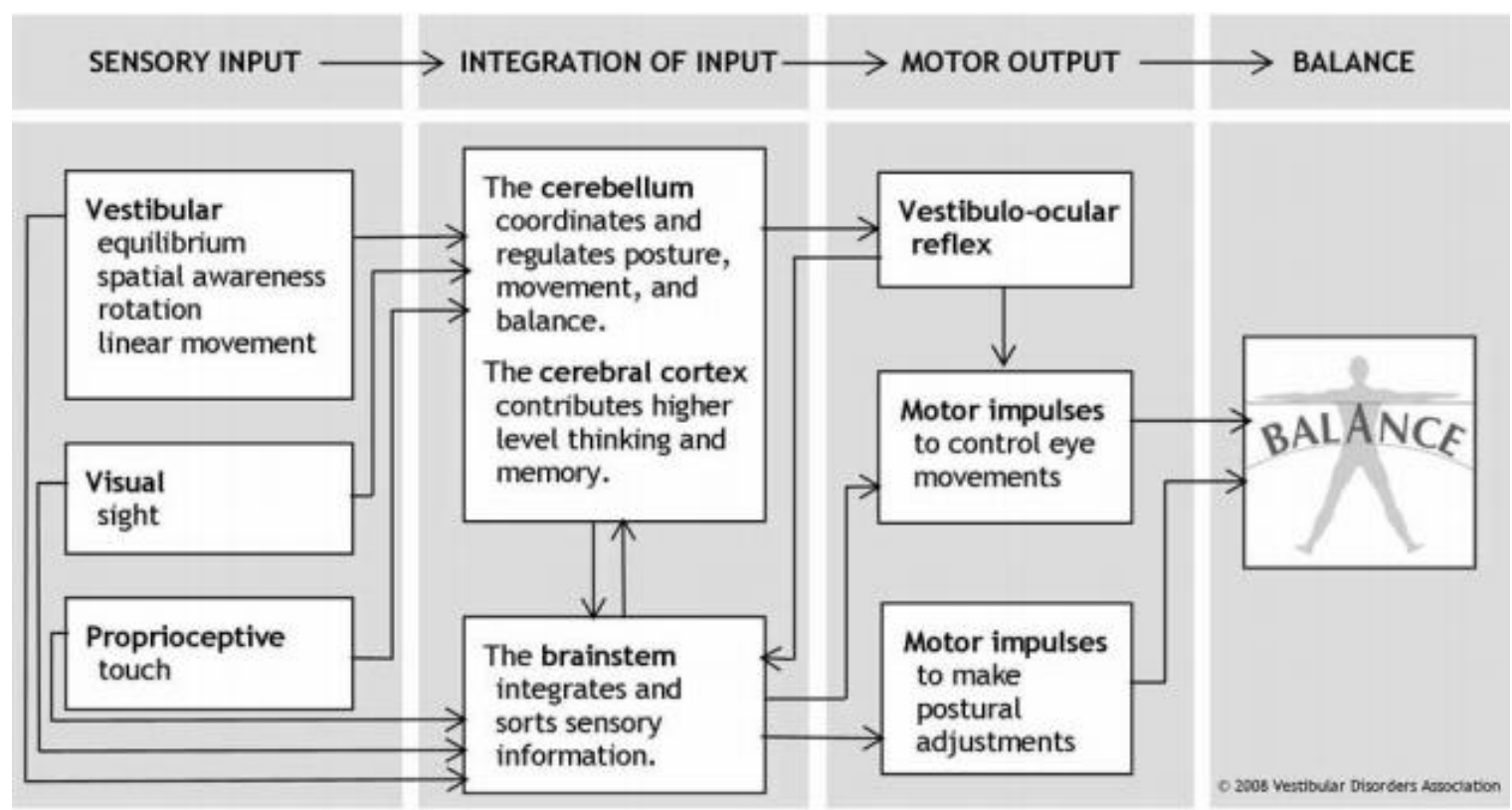

Figure 3. The balance control system (from Vestibular Disorder Association 2008)

Hearing examination, blood tests, an electronystagmogram (a test that measures eye movements and the muscles that control them), or imaging studies of patient head and brain are used for diagnosis of a balance disorder. A test, called posturography, is very popular: patient stands on a special movable platform in front of a patterned screen and the specialist measures how the patient body responds to movement of the platform, the patterned screen, or both.

Figure 4 shows the scheme of a new equipment that has recently been experimented. It is based on a position sensor and an optical sensor whose outputs are received, stored and processed by a computer through an eyetracker algorithm (Qu, \& Purcell, 2015).

Other recent solutions are based on wearable devices. One of the them has been implemented using inertial sensors and sensor fusion processing to measure body posture and provide real time feedback to alert the patient to remain in the region of stability (Hsu et al., 2015).

The effectiveness of current interventions on balance disorder treatments is limited because patients tend to be not fully involved and adhere to the training protocol. Information and communication technologies are opening new opportunities, and many researches are increasing on rehabilitation facilities that simulate real environment (Bower et al., 2014; McEwen et al., 2014). 


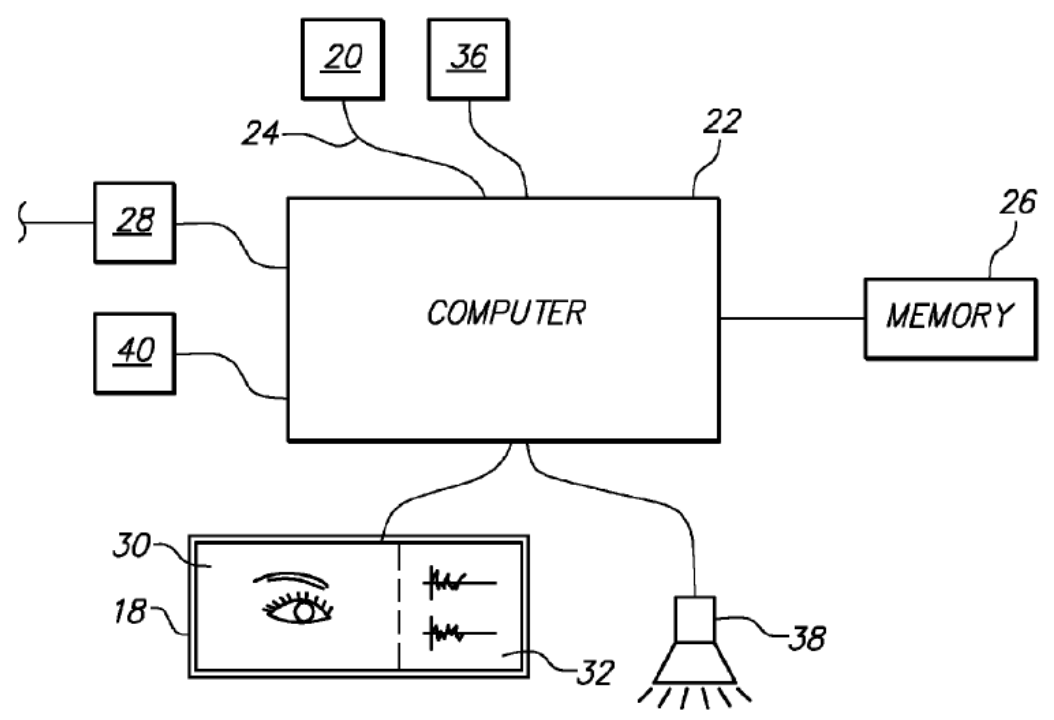

Figure 4. A new equipment for test balance disorders (from Qu, \& Purcell, 2015)

At present, commercial equipment is available to test balance disturbs, and many of them are also used for patient training. Generally, they integrate force platform and video cameras in a computerized system (Browne \& O'Hare, 2001); this kind of equipment was used in PSRI previous research (Figure 5). ${ }^{22}$

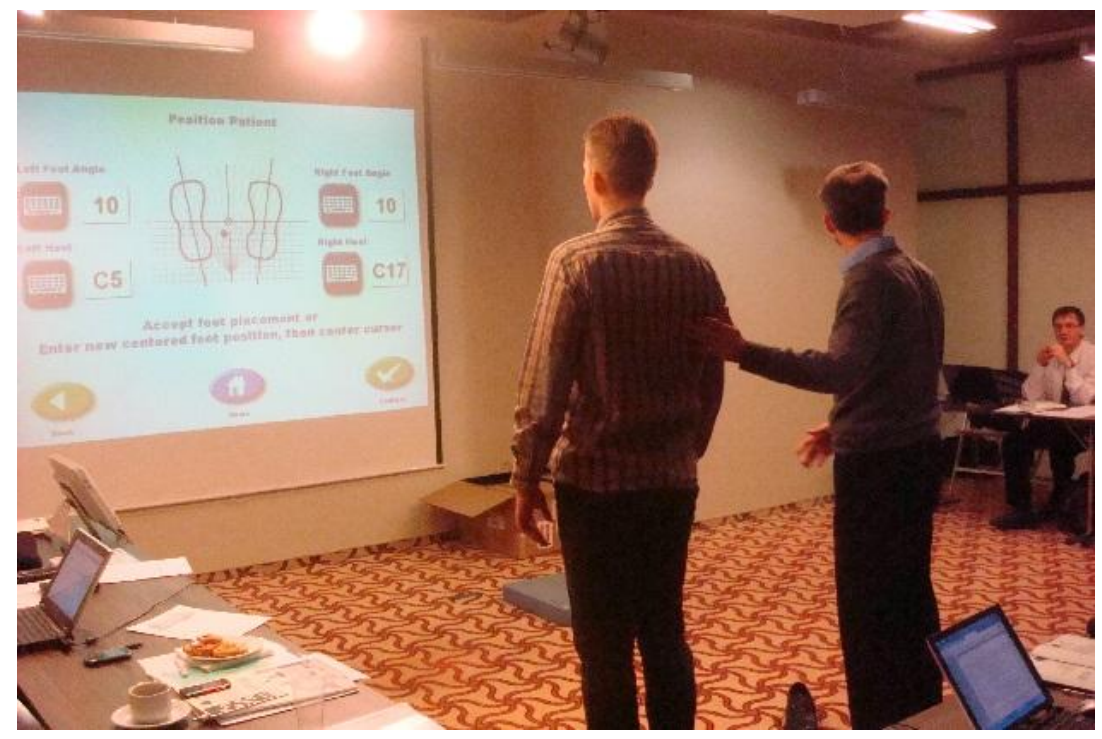

Figure 5. Expertiment in VPP INOSOCTEREHI programme

However, since the aim of our research is social telerehabilitation, our investigation focuses on the social implication of prevention, diagnosis and treatment of balance disorders rehabilitation. There are aspects of rehabilitation that are relational, such as the doctor-patient relationship, the patient rapport with his/her family and his/her work environment. These aspects can negatively

${ }^{22}$ LAT-LIT project "Designing a Model Geared towards Participation of People at Social Risk Groups in the 
affect the results of rehabilitation. Moreover, the use of information and communication technology entails the problem of digital divide; it is physical (lack of connectivity), cultural (not adequate skills to dispense services and/or use them), social (prejudices and biases), economic (not sustainable costs). In addition, one must keep in mind that, without the presence of medical staff, patients miss out something, that could be important. Nevertheless, patients are often able to make more progress at home with the support of their family or people close to them. All the above issues are context sensitive, depending from the specific disease and its rehabilitation process.

The goal of VPP INOSOCTEREHI research programme about telerehabilitation of balance disorders is to highlight its social implication, such as educational requirements and activities that should lead and support telerehabilitation process in order to ensure its feasibility and sustainability.

It implies the need of acquiring a theoretical and practical thorough knowledge, about rehabilitation technology used for balance disorders. Such knowledge is necessary for the definition of distance rehabilitation services, especially at home. Real benefits, problems and limitations of technology should be highlighted, and the greatest attention should be placed on how to minimize and overcome shortages and disadvantages tied to the social sphere. Suggestions and recommendations could be useful to improve the technology, adapting it to actual social environments.

\section{Conclusions}

VPP INOSOCTEREHI research programme is just at the beginning. However, some elements already appear evident, after the scanning of the principal social rehabilitation services in Latvia and the first activities carried out by the project participants.

The creation of social tele-rehabilitation services is not easy. The availability of technology is necessary but not sufficient for the realization of effective social telerehabilitation services. Technological solutions have to be dropped in different contexts, which may vary depending on the different rehabilitation branches. Moreover, delivering rehabilitation services at distance, on the one hand reduces the cost of mobility, but on the other hand can create new relational and control problems. However, the analysis of the current situation shows that information and communication technologies are little used in social rehabilitation. Although their potential is enormous, the specificity of social rehabilitation sectors shows the impossibility of defining general purpose social telerehabilitation solutions.

At the moment, our opinion is that advantages coming from telerehabilitation and its efficacy cannot be generalized and assumed without proof. It would be necessary to investigate the real feasibility of telerehabilitation focusing on specific telerehabilitation services, and define 
models for evaluating their long-term advantages, cost effectiveness, possible improvement in quality of life and impact on public health burden.

The preliminary project activities gave us the opportunity to better define the scope of social telerehabilitation, clarifying its specificity and its interrelations with other branches (telemedicine and telecare), and this is a first basic step in our research.

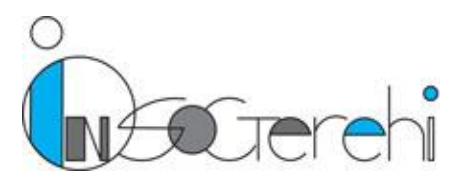

This work has been supported by National Research Program (NRP) "Innovative solutions for social tele-rehabilitation in the schools of Latvia in the context of inclusive education" INOSOCTEREHI

\section{References}

Baranauskienė, I., Gerulaitis, D., Lubkina, V., Radzevičienè, L., Usca, S. (2013). Health Promotion and Professional rehabilitation Technologies for Participation in Labor Market. Rēzekne, Rēzeknes Augstskola.

Bower, K. J., McGinley, J. L., Miller, K. J., \& Clark, R. A. (2014). Instrumented Static and Dynamic Balance Assessment after Stroke Using Wii Balance Boards: Reliability and Association with Clinical Tests. PloS one, 9(12), e115282.

Browne, J. E., \& O'Hare, N. J. (2001). Review of the different methods for assessing standing balance. Physiotherapy, 87(9), 489-495.

Di Cerbo, A., Morales-Medina, J. C., Palmieri, B., \& Iannitti, T. (2015). Narrative review of telemedicine consultation in medical practice. Patient preference and adherence, 9, 65.

Freedman, M. J., Lester, K. M., McNamara, C., Milby, J. B., \& Schumacher, J. E. (2006). Cell phones for ecological momentary assessment with cocaine-addicted homeless patients in treatment. Journal of substance abuse treatment, 30(2), 105-111.

Gorst, S. L., Armitage, C. J., Brownsell, S., \& Hawley, M. S. (2014). Home telehealth uptake and continued use among heart failure and chronic obstructive pulmonary disease patients: A systematic review. Annals of Behavioral Medicine, 48(3), 323-336.

Haig, A. J. (2013). Disability Policy Must Espouse Medical as well as Social Rehabilitation. Social Inclusion, 1(2), 136-138.

Hailey, D., Roine, R., Ohinmaa, A., \& Dennett, L. (2011). Evidence of benefit from telerehabilitation in routine care: a systematic review. Journal of telemedicine and telecare, 17(6), 281-287.

Hill, A. J. (2010). Report on the potential application of telerehabilitation to adult rehabilitation services in Scotland. Scottish Government, Edinburgh.

Hsu, Y., Payson, E., Sapir, I., \& Villacorta, V. (2015). U.S. Patent No. 20,150,018,724. Washington, DC: U.S. Patent and Trademark Office.

Luxton, D. D., McCann, R. A., Bush, N. E., Mishkind, M. C., \& Reger, G. M. (2011). mHealth for mental health: Integrating smartphone technology in behavioral healthcare. Professional Psychology: Research and Practice, 42(6), 505.

Nilsson, A. G., \& Nilsson, T. H. (2011a). Towards a New Concept for Supporting Needy Children in Developing Countries-ICT Centres Integrated with Social Rehabilitation. In Information Systems Development (pp. 437-448). Springer New York.

Nilsson, A. G., \& Nilsson, T. H. (2011b). Global Development and ICT for Building Civil Societies in Developing Countries. In Proceedings of IDIA2011, the $5^{\text {th }}$ International Development Informatics Conference on ICT for Development: People, Policy and Practice, 26-28 October, Lima, Peru. IDIA, Monash University. 
Osmani, V., Maxhuni, A., Grünerbl, A., Lukowicz, P., Haring, C., \& Mayora, O. (2013, December). Monitoring activity of patients with bipolar disorder using smart phones. In Proceedings of International Conference on Advances in Mobile Computing \& Multimedia (p. 85). ACM.

McEwen, D., Taillon-Hobson, A., Bilodeau, M., Sveistrup, H., \& Finestone, H. (2014). Virtual Reality Exercise Improves Mobility After Stroke An Inpatient Randomized Controlled Trial. Stroke, 45(6), 1853-1855.

Mitenbergs, U., Taube, M., Misins, J., Mikitis, E., Martinsons, A., Rurane, A., Quentin, W. (2012). Latvia: Health system review. Health Systems in Transition 2012, 14(8): 1 - 191.

Qu, Y., \& Purcell, I. (2015). U.S. Patent No. 20,150,005,587. Washington, DC: U.S. Patent and Trademark Office.

Rice, E., Kurzban, S., \& Ray, D. (2012). Homeless but connected: the role of heterogeneous social network ties and social networking technology in the mental health outcomes of street-living adolescents. Community mental health journal, 48(6), 692-698.

Rogante, M., Grigioni, M., Cordella, D., \& Giacomozzi, C. (2010). Ten years of telerehabilitation: A literature overview of technologies and clinical applications. Neuro Rehabilitation, 27(4), 287-304.

Rose, G. L., Skelly, J. M., Badger, G. J., Naylor, M. R., \& Helzer, J. E. (2012). Interactive voice response for relapse prevention following cognitive-behavioral therapy for alcohol use disorders: a pilot study. Psychological services, 9(2), 174.

Shumway-Cook, A., \& Woollacott, M. H. (1995). Motor control: theory and practical applications. Lippincott Williams \& Wilkins.

Simpson, T. L., Kivlahan, D. R., Bush, K. R., \& McFall, M. E. (2005). Telephone selfmonitoring among alcohol use disorder patients in early recovery: a randomized study of feasibility and measurement reactivity. Drug and alcohol dependence, 79(2), 241250 .

Terauda, V. A., Reetz, A., Jahn, D. (Coordinator). (2014). Sustainable Governance Indicators, 2014 Latvia report, Bertelsmann Stiftung.

Wang, Q., Chen, W., \& Markopoulos, P. (2014, June). Literature review on wearable systems in upper extremity rehabilitation. In Biomedical and Health Informatics (BHI), 2014 IEEE-EMBS International Conference on (551-555). IEEE.

Winters, J. M. (2002). Telerehabilitation research: emerging opportunities. Annual Review of Biomedical Engineering, 4(1), 287-320. 